\title{
Orthogonal logic gates
}

A set of 16 TetR proteins specific for their synthetic promoters will allow the construction of complex genetic circuits.

Christopher Voigt at the Massachusetts Institute of Technology has a long-standing interest in building genetic circuits to program bacterial cell behavior. Until 2009 his team, like many others in the field, used two or three transcription factors for circuit design, but they realized that to "build the type of circuits we really wanted, we were going to have to bite the bullet and do the hard work to fully characterize orthogonal transcription factors."

Genetic logic gates that make up these circuits often use promoters as input and output signals. A transcription factor acts on an input promoter that drives the expression of an activator or repressor for a downstream, output promoter. For the circuit to work, it is important that a transcription factor is specific to only its designated promoter.
Brynne Stanton, a postdoctoral fellow in the Voigt lab, focused on homologs of the tetracycline repressor (TetR) proteins. These transcriptional repressors are known for their specific binding to operator sequences.

Stanton started by synthesizing 73 TetR proteins from different prokaryotic species and defining their operators with an in vitro array assay. She then constructed promoter libraries in which the specific operator sequences for 20 TetRs were inserted at different positions in a strong Escherichia coli promoter. A screen for specificity showed 16 TetRs with minimal cross-reactivity and with highly diverse operator sequences.

Alec Nielsen, a graduate student in the lab, whom Voigt refers to as the 'circuit-building guru', then used these repressors and promoters to build a library of NOT gates (the output, here yellow fluorescent protein, is off if the input, inducible TetR, is on) and evaluated the dynamic range of each gate. By combining NOT gates in series, the researchers created NOR gates and then used these for simple circuits with two Boolean logic operations: AND (output is on only if both inputs are present) or NAND (output is off only in the presence of both inputs). A remaining challenge is to ensure that the gates will function reliably in any genetic context. "The gates subtly change their response function when put into different locations," says Voigt. "This can cause the construction of larger circuits to fail."

Also, an automated process for circuit design is needed. Together with computational experts, Voigt is working on the first programming language for synthetic biology.

\section{Nicole Rusk}

\section{RESEARCH PAPERS}

Stanton, B.C. et al. Genomic mining of prokaryotic repressors for orthogonal logic gates. Nat. Chem. Biol. 10, 99-105 (2014). 